\title{
Marital Status Independently Predicts Survival in Patients with Small Cell Lung Cancer: A SEER Database Analysis
}

\section{Pei Luo ( $\square$ 2727635154@qq.com )}

The Seventh Affiliated Hospital of Zunyi Medical University (Qianxinan People's Hospital)

\section{Yan Mao}

Zunyi medical university \Zunyi 563000

\section{Liping Yang}

The Seventh Affiliated Hospital of Zunyi Medical University (Qianxinan People's Hospital)

\section{Chao Pan}

The Seventh Affiliated Hospital of Zunyi Medical University (Qianxinan People's Hospital)

\section{Jun Guo}

The Seventh Affiliated Hospital of Zunyi Medical University (Qianxinan People's Hospital)

\section{Research Article}

Keywords: Marital status, Small cell lung cancer, SEER, Overall survival

Posted Date: September 8th, 2021

DOI: https://doi.org/10.21203/rs.3.rs-832341/v1

License: (c) (i) This work is licensed under a Creative Commons Attribution 4.0 International License. Read Full License 


\section{Abstract}

Purpose This study will investigate the relationship between marital status and prognosis in small cell lung cancer patients.

Methods Patients of SCLC was selected from the SEER database (1973-2013) and the patient sinformation. Kaplan-Meier analysis, log-rank test and Cox regression model were used for studying patientprognosis.

Result 27069 SCLC patients eligible for inclusion were screened from the SEER database. Kaplan-meier test showed that the median OS values were 8, 7, 6 months in married, single and SDW patients, respectively.

Conclusion This study shows that marital status is an independent prognostic factor for overall survival in SCLC patients. Married patients with small cell lung cancer have better prognosis than those who were divorced/separated, widowed and single.

\section{Introduction}

Lung cancer is the most common malignant tumor in the world and the number one cause of cancer death ${ }^{[1]}$. Among them, small cell lung cancer (SCLC) accounts for $15-20 \%$ of the total number of newly diagnosed lung cancers in the world each year ${ }^{[2]}$. Since SCLC is the least differentiated but most malignant type in lung cancer, it has a short doubling time, high blood vessel density, and genomic instability Compared to non-small cell lung cancer, the characteristics of stable and extremely distant metastasis have shown little improvement in SCLC detection, treatment and survival in the past 30 years $^{[3,4]}$. If no measures are taken for treatment, the median survival time is generally only 2 to 4 months After comprehensive treatment, the median survival time for patients with limited period can reach 15-20 months ${ }^{[5,6]}$. The 2 -year survival rate is $20 \%-40 \%$, and the median survival period for patients with extensive stage can reach $7-13$ months, about $5 \%$ reaches 2 years ${ }^{[7]}$. Facing the grim situation, we are urged to continuously increase our research on SCLC.

As an important factor affecting human physical and mental health and quality of life, the quality of marriage has received increasing attention ${ }^{[8,9]}$. Some studies have shown that harmonious marriage can prolong the life of cancer patients ${ }^{[10]}$. About 750,000 patients with different types of tumors were selected,. These tumors include colon cancer, prostate cancer, etc., it was found that before the tumor appeared to metastasize, tumor patients with a harmonious and happy marriage had a $20 \%$ reduction in the probability of dying from cancer ${ }^{[11]}$. A harmonious marriage could improve the survival rate of tumor patients. After analyzing relevant data from 127,753 patients with colon cancer, the researchers found that married patients diagnosed at earlier period had mortality rate $14 \%$ lower than that of unmarried, widowed or divorced patients ${ }^{[12]}$. The exact mechanism of how marriage plays a positive role in colon cancer diagnosis and treatment is not fully understood ${ }^{[13]}$. The spouse can play a key positive role in the 
disease, and this effect is conducive to the improvement of survival rate $\mathrm{e}^{[14,15]}$. However, there are no reports about the relationship between marital status and prognosis in patients with small cell lung cancer. In this study, we performed a comprehensive population-based analysis using the SEER database to clarify the prognostic significance of marital status for SCLC patient survival.

\section{Data And Methods}

\subsection{Study Population And Data Collection}

Patients diagnosed with small cell lung cancer from 1973 to 2013 in the American Surveillance, Epidemiology, and End Results (SEER) database were selected. The data was analyzed using the SEER database (version 8.3.2, updated April 14, 2016).

\section{The inclusion criteria:}

(i) Patient was histologically confirmed with small cell lung cancer (ICD-0-3 histology code: 8041, 8042, $8043,8044)$

(ii) Patient was over 18 years old

(iii) Full survival follow-up time

\section{The exclusion criteria:}

(i) Patients with nonprimary SCLC

(ii) Missing marital status data

(iii) Patient baseline information was incomplete, such as sex, race and age

(iv) Tumor information (histology grade) was not recorded

Patient treatment information (radiotherapy and surgery) was unclear

\subsection{Methods}

The case-control studies were used to divide patients into married group, single group, and SDW groups (divorced, separated, widowed) based on their marital status, the outcome index was overall survival (OS). The variables included in this study were sex, marital status, age, race, tumor classification, surgical status, and radiotherapy status. Marital status was divided into three groups: married group, single group and SDW group (divorced, separated, widowed). Race was divided into white group, black group and 
other races group. Age is divided into various age groups. The tumors histological grades were divided into I group, II group, III group and IV group. According to whether surgery is divided into two groups: surgery and non-operation; according to whether radiotherapy is divided into two groups: radiotherapy and non-radiotherapy.

\subsection{Statistical methods}

The data was extracted by Perl 5.26 .2 software, data were collated with Excel 2013 software, statistical analysis was performed with SPSS 23.0 and R 3.35 software, chi-square test was used for patient baseline characteristics test, Kaplan-Meier and Log rank test were used for single factor survival efficiency analysis and survival curve was drawn, Cox multivariate analysis study The effect of each variable on the survival prognosis of lung cancer patients, $P<0.05$ was statistically significant.

\section{Results}

\subsection{Baseline characteristics}

A total of 27069 eligible SCLC patients aged from 18 to 98 were included during the study period (from 1973 to 2013$)$ in the SEER database, with an average age of $(67.71 \pm 10.32)$ years old. Among them, 14451 (53.39\%) were married, 3167(11.70\%) were never married, 9451 (34.91\%) were in SDW group, which included 4955 (52.43\%) widowed, 3706 (39.21\%) divorced, and 790 (8.36\%) separated. Age, race, histological grade, radiotherapy and surgery were different in marital composition $(P<0.01)$, as shown in Table 1.

\subsection{Relationship between patient characteristics and survival prognosis}

Kaplan-meier analysis showed that age, race, marital status, histological grade, radiotherapy and surgery were all related to the survival prognosis of patients. The prognosis of patients with bad marriage is significantly worse than that of married patients. There were statistically significant differences in prognosis between patients of different genders. There was significant difference in prognosis among different races $(P<0.01)$. The prognosis of patients $<65$ years old was significantly better than that of patients $\geq 65$ years old. The prognosis of the patients after radiotherapy was significantly better than that of the patients without radiotherapy; The difference of prognosis in different histological stages was statistically significant $(P<0.01)$. The prognosis of patients undergoing surgery was significantly better than that of patients without surgery, as shown in Figure A1-7.

In order to remove the influence of confounding factors, the above analysis factors with $P<0.05$ were included in the COX regression model for multivariate analysis. The results showed: age, race, marital 
status, histological grade, radiotherapy or not and surgery or not were independent factors of survival prognosis in SCLC, as shown in Table 2 and multivariate Cox forest map(Figure B).

\subsection{Subgroup analysis based on survival rate}

In order to exclude the influence of all other possible factors on the ultimate survival, we did a stratified analysis to determine the independent prognostic effect of marital status on small cell lung cancer patients. Kaplan-Meier survival curves stratified by sex, age (cut-off value of 65 years), radiotherapy and surgery were shown in Figures A8-11, respectively. Subgroup analysis stratified by sex, age and radiotherapy displayed that married group had better survival compared with patients in SDW and single groups(Figures A8-10). Figure A11 depicted that married people had longer survival compared with those in SDW and single groups when individually analyze data from patients not receiving surgery.

\section{Discussion}

The results of this study showed that marital status is an independent prognostic factor for patients with small cell lung cancer. SCLC patients in Single group and SDW group had poor prognosis and a higher risk of death. Therefore, the inharmonious marital state is one of the risk factors for poor prognosis of small cell lung cancer. Without the companion, it may easily lead to the change of patients' mood, affect their quality of life, change their role function, and cause serious adverse effects on their psychological state $^{[16,17]}$ Studies have found that mental state can affect the progress of tumor, aggravate the clinical symptoms of patients, and then affect the clinical prognosis ${ }^{[18]}$. Positive emotions have a buffering effect on stress, can alleviate the psychological burden, stimulate patients to take positive behaviors, and significantly improve the psychological state and prognosis of cancer patients ${ }^{[19]}$. However, negative emotions may induce changes in the respiratory system and cardiovascular system, which may lead to poor prognosis ${ }^{[20]}$.

The researchers conducted a large epidemiological survey and analyzed data from 1,260,898 patients with various types of tumors, such as prostate cancer, breast cancer, lymphoma, colorectal cancer, pancreatic cancer, ovarian cancer, esophageal cancer, lung cancer, head and neck tumors, etc ${ }^{[21,22]}$. The results showed that after correction for all confounding factors, married cancer patients had a lower risk of death from cancer. Researchers have analyzed that cancer patients in happy families were more likely to receive health checkups recommended by their family members, so that they can be diagnosed and treated early in the disease ${ }^{[23]}$. At the same time, after being diagnosed, these patients are more willing to strictly follow the treatment plan and conduct the follow up supervision regularly. The results show social support is important for cancer patients ${ }^{[24]}$.

Cancer has an impact on both physiology and psychology of patients. For example, the clinical symptoms of the disease will bring physical pain to patients, and the fear of disease recurrence and death will make patients suffer from psychological loneliness and depression ${ }^{[25]}$. Social support is 
considered to be that individuals receive support from the outside world, including material and spiritual guarantees, which directly promote the physical and mental health of individuals Study showed that social support can inhibit the progress of cancer ${ }^{[26]}$. Compared with the control group, the survival rate of the group participating in social support was significantly improved. Marriage is a form of social support. Social support in marriage mainly comes from the spouse. The patient's spouse can provide effective emotional comfort and social environment support in time to reduce the psychological stress of the patient ${ }^{[27]}$. Therefore, single or SDW patients with SCLC should be provided with active psychological counseling. The doctors should answer patient questions patiently, communicate in a timely manner and give psychological intervention to reduce the negative emotions of patients, so as to improve their quality of life and prolong their survival time. It is also suggested for the medical staff to give patients more humane care and emotional support while treating tumors, this will reduce their psychological pain, improve their internal control, and then effectively improve their psychological state ${ }^{[28]}$.

The SEER database provides us the opportunity to perform large, population-based studies. However, there are several limitations in our study. An obvious limitation is that this is a retrospective study that has a greater probability of bias than prospective studies. In addition to demographic and clinical factors, there is a relative lack of control variables. For example, the database cannot provide important confounding variables such as smoking history, patient complications, and other treatment options. Secondly, the marital status recorded in the SEER database is only the marital status at the time of diagnosis. As for whether the patient's subsequent marital status has changed, no follow-up visits and records have been performed, so the impact of this change on the study cannot be estimated. Thirdly, the SEER database does not incorporate data on the life quality of married patients, such as marital satisfaction or financial status.

Despite of these potential limitations, this is the first study investigating the effect of marital status on prognosis of SCLC by big data. It suggests that doctors should pay more attention to unmarried or SDW patients. This will improve the mental health problems of such patients caused by the lack of companion, thus achieve better clinical treatment measures and improve the long-term prognosis.

\section{Abbreviations}

SEER:Surveillance Epidemiology and End Results

SCLC:Small Cell Lung Cancer

OS: Overall Survival

SDW: Divorced, Separated, Widowed 
HR: Hazard ratio

\section{Declarations}

\section{Ethics approval}

All procedures in studies involving human participants were performed in accordance with the ethical standards of the institutional review board of the second affiliated hospital of zunyi medical university Ethics Committee basing on the 1964 Helsinki declaration and its later amendments.

\section{Consent for publication}

Not applicable.

\section{Acknowledgements}

The authors thank the reviewers for their helpful comments on this article.

\section{Funding}

No.

\section{Availability of data and materials}

The dataset generated and analyzed during the current study is available in the Surveillance Epidemiology and End Results (SEER) Database repository [https://seer.cancer.gov/].

\section{Authors' contribution}

PL provided contribution to (i) data analysis and interpretation and (iii) manuscript drafting and critical revising. PL and YM contributed to (i) experimental design, (ii) data analysis and interpretation and (iii) critical manuscript revising for important intellectual content. LP-Y and CP provided contribution to (i) data analysis and (ii) critical manuscript revising for important intellectual content. JG contributed to (i) data analysis and interpretation and (ii) critical manuscript revising for important intellectual content. All authors have approved the final version and submission of this manuscript.

\section{Competing interests}


The authors declare no conflicts of interest in preparing this article.

\section{References}

1. Siegel, R. L., Miller, K. D. \& Jemal, A. Cancer statistics, 2020[J]. CA Cancer J Clin, 70 (1), 7-30 (2020).

2. Rudin, C. M. et al. Small-cell lung cancer[J]. Nat Rev Dis Primers, 7 (1), 3 (2021).

3. Wang, Y. et al. New insights into small-cell lung cancer development and therapy[J]. Cell Biol Int, 44 (8), 1564-1576 (2020).

4. Islami, F., Torre, L. A. \& Jemal, A. Global trends of lung cancer mortality and smoking prevalence[J].Transl Lung Cancer Res.2015, 4(4):327-338

5. Peng, A. et al. Role of surgery in patients with early stage small-cell lung cancer[J]. Cancer Manag Res, 11, 7089-7101 (2019).

6. Yang, Y. et al. Benefits of surgery in the multimodality treatment of stage iib-iiic small cell lung cancer[J]. J Cancer, 10 (22), 5404-5412 (2019).

7. Zhao, X. et al. Surgical resection of sclc: Prognostic factors and the tumor microenvironment[J]. $J$ Thorac Oncol, 14 (5), 914-923 (2019).

8. Hofman, A. et al. Improving social support to increase qol in lung cancer patients[J]. Cancer Manag Res, 13, 2319-2327 (2021).

9. Lan, T. et al. Effects of marital status on prognosis in women with infiltrating ductal carcinoma of the breast: A real-world 1: 1 propensity-matched study[J]. Med Sci Monit, 26, e923630 (2020).

10. Aizer, A. A. et al. Marital status and survival in patients with cancer[J]. J Clin Oncol, 31 (31), 38693876 (2013).

11. Hanske, J. et al. The influence of marital status on the use of breast, cervical, and colorectal cancer screening[J]. Prev Med, 89, 140-145 (2016).

12. Li, Q. et al. The influence of marital status on stage at diagnosis and survival of patients with colorectal cancer[J].Oncotarget.2015, 6(9):7339-7347

13. Feng, L. et al. Marital status and survival of patients with colorectal signet ring cell carcinoma: A population-based study[J]. Sci Rep, 10 (1), 17881 (2020).

14. Rendall, M. S. et al. The protective effect of marriage for survival: A review and update[J].Demography.2011, 48(2):481-506

15. Liu, Y. L. et al. Marital status is an independent prognostic factor in inflammatory breast cancer patients: An analysis of the surveillance, epidemiology, and end results database[J]. Breast Cancer Res Treat, 178 (2), 379-388 (2019).

16. Tatangelo, G. et al. Gender, marital status and longevity[J].Maturitas.2017, 100:64-69

17. Wu, Y., Ai, Z. \& Xu, G. Marital status and survival in patients with non-small cell lung cancer: An analysis of 70006 patients in the seer database[J].Oncotarget.2017, 8(61):103518-103534 
18. Applebaum, A. J. et al. Optimism, social support, and mental health outcomes in patients with advanced cancer[J]. Psychooncology, 23 (3), 299-306 (2014).

19. Dunstan, D. A. \& Scott, N. Norms for zung's self-rating anxiety scale[J]. BMC Psychiatry, 20 (1), 90 (2020).

20. Hu, T. et al. Relationship between resilience, social support as well as anxiety/depression of lung cancer patients: A cross-sectional observation study[J]. J Cancer Res Ther, 14 (1), 72-77 (2018).

21. Fitzmaurice, C. et al. Global, regional, and national cancer incidence, mortality, years of life lost, years lived with disability, and disability-adjusted life-years for 29 cancer groups, 1990 to 2017: A systematic analysis for the global burden of disease study[J]. JAMA Oncol, 5 (12), 1749-1768 (2019).

22. Henley, S. J. et al. Annual report to the nation on the status of cancer, part ii: Progress toward healthy people 2020 objectives for 4 common cancers[J]. Cancer. 2020, 126(10): 2250-2266

23. Chen, Z. H. et al. Assessment of modifiable factors for the association of marital status with cancerspecific survival[J].JAMA Netw Open.2021, 4(5):e2111813

24. Osborne, C. et al. The influence of marital status on the stage at diagnosis, treatment, and survival of older women with breast cancer[J]. Breast Cancer Res Treat, 93 (1), 41-47 (2005).

25. Luszczynska, A. et al. Emotional support for men and women with cancer: Do patients receive what their partners provide?[J]. Int J Behav Med, 14 (3), 156-163 (2007).

26. Aydın, S. Ä. A. \& Demir Doğan, M. Illness perception, perceived social support and quality of life in patients with diagnosis of cancer[J].Eur J Cancer Care (Engl).2020, 29(4):e13252

27. Gao, Z. et al. Marital status and survival of patients with chondrosarcoma: A population-based analysis[J]. Med Sci Monit, 24, 6638-6648 (2018).

28. Yan, X. R., Chen, X. \& Zhang, P. Prevalence and risk factors of depression in patients with lung cancer: Protocol for a systematic review and meta-analysis[J].BMJ Open.2019, 9(8):e028994

\section{Tables}

Due to technical limitations, tables are only available as a download in the Supplemental Files section.

\section{Figures}




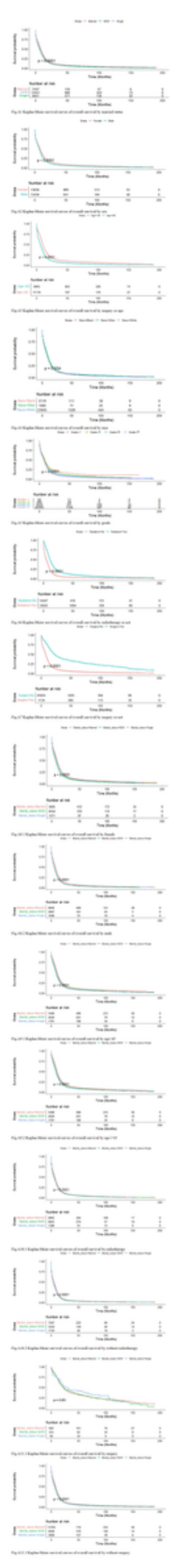

\section{Figure 1}

A) The prognosis of patients undergoing surgery was significantly better than that of patients without surgery, as shown in Figure A1-7. In order to remove the influence of confounding factors, the above analysis factors with $\mathrm{P}<$ Kaplan-Meier survival curves stratified by sex, age (cut-off value of 65 years), radiotherapy and surgery were shown in Figures A8-11, respectively. Subgroup analysis stratified by sex, age and radiotherapy displayed that married group had better survival compared with patients in SDW 
and single groups(Figures A8-10). Figure A11 depicted that married people had longer survival compared with those in SDW and single groups when individually analyze data from patients not receiving surgery. B)multivariate Cox forest map

\section{Supplementary Files}

This is a list of supplementary files associated with this preprint. Click to download.

- Table101.jpg

- Table201.jpg 